\title{
Probing for Bridging Inference in Transformer Language Models
}

\author{
Onkar Pandit \\ University of Lille, INRIA Lille, \\ CNRS, Centrale Lille,UMR 9189-CRIStAL, \\ F-59000, Lille, France \\ onkar.panditeinria.fr
}

\author{
Yufang Hou \\ IBM Research Europe \\ Dublin, Ireland \\ yhoulie.ibm.com
}

\begin{abstract}
We probe pre-trained transformer language models for bridging inference. We first investigate individual attention heads in BERT and observe that attention heads at higher layers prominently focus on bridging relations incomparison with the lower and middle layers, also, few specific attention heads concentrate consistently on bridging. More importantly, we consider language models as a whole in our second approach where bridging anaphora resolution is formulated as a masked token prediction task (Of-Cloze test). Our formulation produces optimistic results without any finetuning, which indicates that pre-trained language models substantially capture bridging inference. Our further investigation shows that the distance between anaphor-antecedent and the context provided to language models play an important role in the inference.
\end{abstract}

\section{Introduction}

Bridging inference involves connecting conceptually related discourse entities - anaphors and antecedents (Clark, 1975). A bridging anaphor shares non-identical relation with its antecedent and depends on it for complete interpretation. This differs from coreference resolution which links mentions that refer to the same entity (i.e., mentions in the same entity share identical relations). Consider the following example -

"In Poland's rapid shift from socialism to an undefined alternative, environmental issues have become a cutting edge of broader movements to restructure the economy, cut cumbersome bureaucracies , and democratize local politics."

Bridging inference connects the anaphor "the economy" and its antecedent "Poland" and deduces that "the economy" specifically refers to "the economy of Poland".

We want to investigate if the pre-trained transformer language models capture any bridging inference information. Recently there has been an in- creasing interest in analyzing pre-trained language models' ability at capturing syntactic information (Clark et al., 2019), semantic information (Kovaleva et al., 2019), as well as commonsense knowledge (Talmor et al., 2020). There are also a few studies focusing on probing coreference information in pre-tained language models (Clark et al., 2019; Sorodoc et al., 2020). So far, there has no work on analyzing bridging, which is an important type of entity referential information. We try to fill this gap in our work.

We employ two different but complementary approaches for the probing of pre-trained transformer language models for bridging inference. In the first approach (Section 4), we investigate the core internal part of transformer models - self-attention heads in vanilla BERT (Devlin et al., 2019). We look at the attention heads of each layer separately and measure the proportion of attention paid from anaphor to antecedent and vice versa. This captures the magnitude of bridging signal corresponding to each attention head. We observed that attention heads of higher layers are more active at attending at bridging relations as well as some of the individual attention heads prominently look at the bridging inference information.

In the second approach (Section 5), we treat pretrained transformer language models as a black box and form bridging inference as a masked token prediction task. This formulation takes into consideration the whole architecture and weights of the model rather than concentrating on individual layers or attention heads, thus, complementing our first approach where we looked at the individual parts of the transformer model. For each bridging anaphor, we provide input as "context anaphor of [MASK]" to language models and get the scores of different antecedent candidates for mask tokens. We then select the highest scoring candidate as the predicted antecedent. Surprisingly, the best variation of this approach produces a high 
accuracy score of $28.05 \%$ for bridging anaphora resolution on ISNotes (Markert et al., 2012) data without any task-specific fine-tuning of the model. On the same corpus, the current state-of-the-art bridging anaphora resolution model BARQA (Hou, 2020a) achieves an accuracy of $50.08 \%$, while a solid mention-entity pairwise model with carefully crafted semantic features (Hou et al., 2013) produces an accuracy score of $36.35 \%$. This shows that substantial bridging information is captured in the pre-trained transformer language models.

Bridging inference requires both commonsense world knowledge as well as context-dependent text understanding. The above-mentioned fill-in-thegap formulation for the antecedent selection task is flexible enough to easily explore the role of different types of context for bridging inference. Our analysis shows that pre-trained language models capture bridging inference substantially however the overall performance depends on the context provided to the model. It is also observed that bigger language models are more accurate at capturing bridging information.

This work has two main contributions. First, we thoroughly investigate bridging information encoded in pre-trained language models using two probing approaches (attention heads analysis and fill-in-the-gap). Second, we provide a deeper understanding of the bridging referential capabilities in the current pre-trained language models. Our experimental code is available at https://github. com/oapandit/probBertForbridging.

\section{Related Work}

Entity Referential Probing. Previous studies on entity referential probing mainly focus on coreference. Clark et al. (2019) showed that certain attention heads in pre-trained BERT correspond well to the linguistic knowledge of coreference. Particularly, the authors found that one of BERT's attention heads achieves reasonable coreference resolution performance compared to a string-matching baseline and performs close to a simple rule-based system. Sorodoc et al. (2020) investigated the factors affecting pronoun resolution in transformer architectures. They found that transformer-based language models capture both grammatical properties and semantico-referential information for pronoun resolution. Recently, Hou (2020b) analyzed the attention patterns of a fine-tuned BERT model for information status (IS) classification and found that the model pays more attention to signals that correspond well to the linguistic features of each IS class. For instance, the model learns to focus on a few premodifiers (e.g., "more", "other", and "higher") that indicate the comparison between two entities. In this work, we focus on probing bridging, which is a more challenging entity referential relation and one of the oldest topics in computational linguistics (Clark, 1975; Bos et al., 1995; Asher and Lascarides, 1998).

Attention Analysis. Recently there has been an increasing interest in analyzing attention heads in transformer language models. Although some researchers argue that attention does not explain model predictions (Jain and Wallace, 2019), analyzing attention weights still can help us to understand information learned by the models (Clark et al., 2019). Researchers have found that some BERT heads specialize in certain types of syntactic relations (Htut et al., 2019). Kovaleva et al. (2019) reported that pre-trained BERT's heads encode information correlated to FrameNet's relations between frame-evoking lexical units (predicates, such as "address") and core frame elements (such as "issues"). In our work, we try to analyze whether certain attention heads in a pre-trained BERT model capture bridging relations between entities in an input text.

Fill-in-the-gap Probing. One of the popular approaches to probe pre-trained language models is fill-in-the-gap probing, in which the researchers have constructed various probing datasets to test a model's ability on different aspects. Goldberg (2019) found that BERT considers subject-verb agreement when performing the cloze task. Petroni et al. (2019) reported that factual knowledge can be recovered surprisingly well from pre-trained language models. For instance, "JDK is developed by [Oracle]". Similarly, we apply fill-in-the-gap to probe bridging by formulating bridging anaphora resolution as a of-Cloze test.

Commonsense Knowledge Probing. A lot of work has been carried out to analyze various types of commonsense knowledge encoded in transformer language models. Talmor et al. (2020) constructed a set of probing datasets and test whether specific reasoning skills are captured by pre-trained language models, such as age comparison and antonym negation. Da and Kasai (2019) found that pre-trained BERT failed to encode some ab- 
stract attributes of objects, as well as visual and perceptual properties that are likely to be assumed rather than mentioned.

In our work, we focus on investigating the effect of context on bridging inference using a wellestablished task on bridging resolution. We extensively analyze the impacts of different contexts for bridging anaphora resolution. We found that a pre-trained BERT model achieves reasonable results for bridging anaphora resolution by using the word "of" as the additional context. This indicates that pre-trained language models capture certain commonsense world knowledge for bridging.

\section{Methodology}

In this paper, we mainly investigate the following research questions:

- How important are the self-attention patterns of different heads for bridging anaphora resolution?

- Whether pre-trained LMs capture information beneficial for resolving bridging anaphora in English?

- How does distance between anaphorantecedent and context influence pre-trained language models for bridging inference?

We designed a series of experiments to answer these questions which will be detailed in the coming sections. In these experiments, we used PyTorch (Wolf et al., 2020) implementation of BERTbase-cased, BERT-large-cased, ROBERTA-base and ROBERTA-large pre-trained transformer language models with the standard number of layers, attention heads, and parameters. In the attention head-based experiments, we have limited our investigation only to the BERT-base-cased model as it is relatively smaller compared to other models and findings of this model can be generalized to other models as well.

Probing Dataset We used ISNotes (Markert et al., 2012) dataset for all experiments. We choose this corpus because it contains "unrestricted anaphoric referential bridging" annotations among all available English bridging corpora (Roesiger et al., 2018) which covers a wide range of different relations. ISNotes contains 663 bridging anaphors but only 622 anaphors have noun phrase
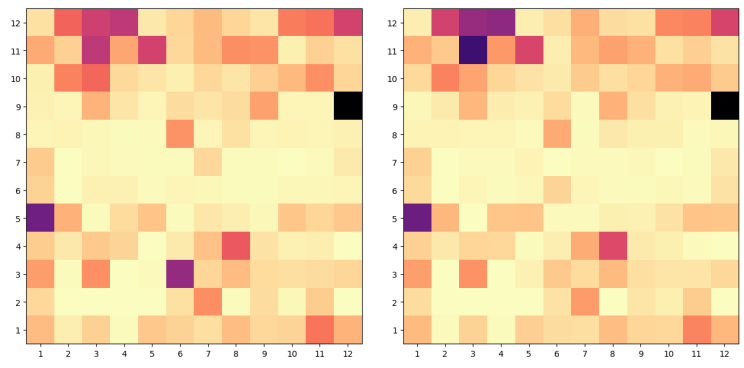

Figure 1: Bridging signals with BERT-base-cased model with only anaphor and antecedent sentences provided. Bridging signals from anaphor to antecedent are shown in the first heatmap and the reverse signals in the second. In both heatmaps, the $\mathrm{x}$-axis shows the attention head number and the $\mathrm{y}$-axis shows the layer number.

antecedents. ${ }^{1}$ In our experiments, we only consider these 622 anaphors for investigation. For any anaphor, the predicted antecedent is selected from the set of antecedent candidates. This set is formed by considering all the mentions which occur before the anaphor. We obtained the candidate set for each anaphor by considering "gold mentions" annotated in ISNotes. Further, we observed that only 531 anaphors have antecedents in either previous 2 sentences from the anaphor or the first sentence of the document. Therefore, in the experiments when antecedent candidates are considered from the window of previous two sentences plus the document's first sentence, only 531 anaphors are considered. In all the experiments, accuracy is measured as the ratio between correctly linked anaphors to the total anaphors used in that particular experiment (not total 663 anaphors).

\section{Probing Individual Attention Heads}

Attention heads are an important part of transformer based language models. Each layer consists of a certain number of attention heads depending on the model design and each attention head assigns different attention weight from every token of the input sentence to all the tokens. In our approach, we measure the attention flow between anaphors and antecedents for each attention head separately. In this experiment we investigate all the attention heads of every layer one-by-one. Specifically, the BERT-base-cased model used for probing contains 12 layers and 12 attention heads at each layer. Therefore, we investigate 144 attention heads for their ability to capture bridging signals.

\footnotetext{
${ }^{1} \mathrm{~A}$ small number of bridging antecedents in ISNotes are represented by verbs or clauses.
} 

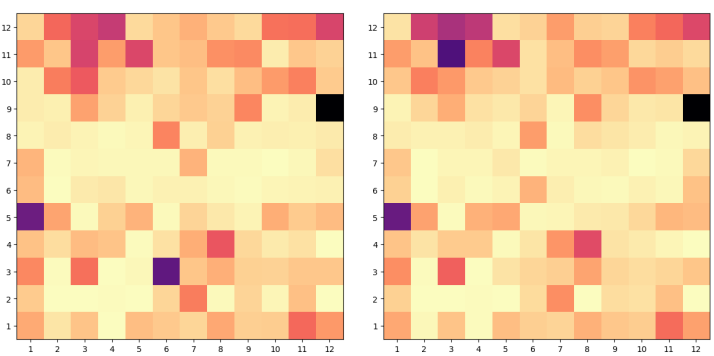

(a) Anaphor-antecedent sent. distance 0
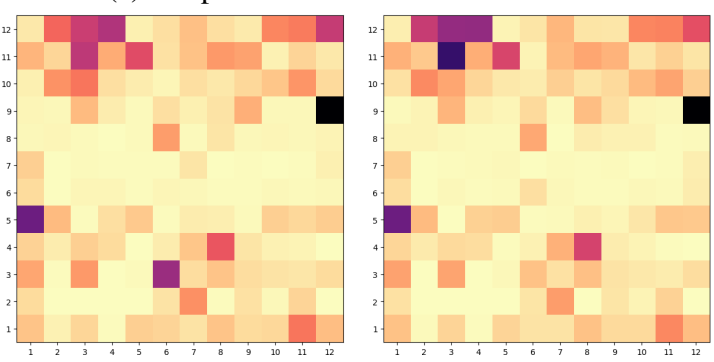

(b) Anaphor-antecedent sent. distance 1
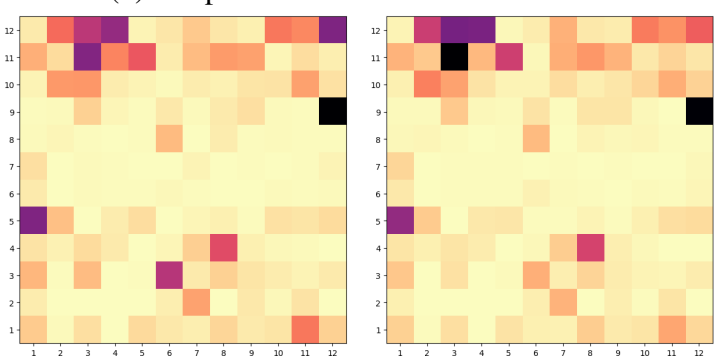

(c) Anaphor-antecedent sent. distance 2

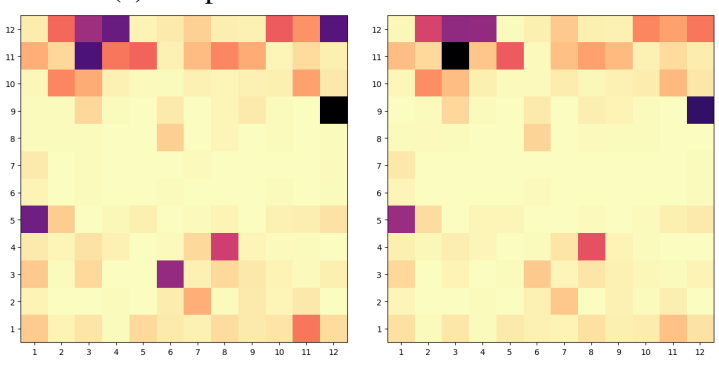

(d) Anaphor-antecedent sent. distance between 3 and 5

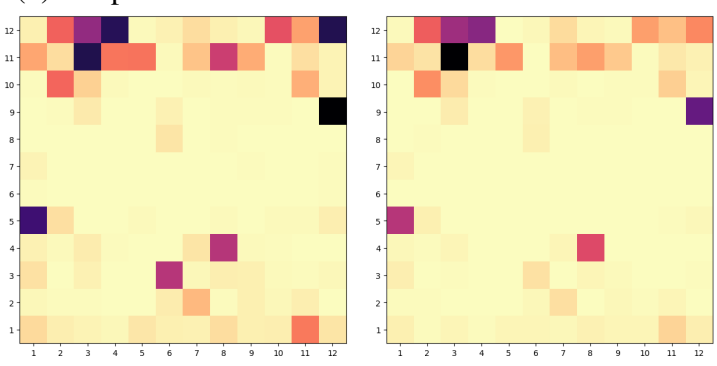

(e) Anaphor-antecedent sent. distance between 6 and 10

Figure 2: Bridging signals in the pre-trained BERT-basecased model with the input including all the sentences between an anaphor and its antecedent. Different heatmaps are shown depending on the sentence distance between anaphor and antecedent. The first heatmap in each row shows the signals from anaphor to antecedent and the second one from antecedent to anaphor. All the heatmaps present the attention heads on the $\mathrm{x}$-axis and the layer numbers on the $\mathrm{y}$-axis.

\subsection{Bridging Signal}

We look for two distinct bridging signals - one from anaphor to antecedent and other from antecedent to anaphor. The bridging signal from anaphor to antecedent is calculated as the ratio of the attention weight assigned to antecedent and the total cumulative attention paid to all the words in the input. Similarly, the bridging signal from antecedent to anaphor is found in a reverse way.

There are two difficulties while getting the attention weights corresponding to anaphor or antecedent. First, the anaphor or antecedent can be a phrase with multiple words. So, we need to decide how to aggregate words' weights. For this, we decide to consider the semantic heads of both anaphor and antecedent, and get the attention weight between them. For instance, the semantic head for "the political value of imposing sanction against South Africa" is "value". Most of the time, a semantic head of an NP is its syntactic head word as in the above example. However, for coordinated NPs such as "the courts and the justice department", the syntactic head will be "and" which does not reflect this NP's semantic meaning. In such cases, we use the head word of the first element as its semantic head (i.e., courts).

Secondly, transformer language models use the wordpiece tokenizer to break words further. This produces multiple tokens from a single word if this word is absent from the language model's dictionary. Here, for a bridging anaphor $a$ and its head word $a_{h}$, we first calculate the average weight of all word piece tokens of the head word $a_{h}$ to other words. From these weights, we consider the weight from the anaphor $a$ to its antecedent $\left(w_{1}\right)$. Subsequently, we add weights from $a_{h}$ to all other tokens present in the sentence and normalize the weight using sentence length $\left(w_{2}\right)$. Note that we neglected weights assigned to special tokens (i.e. [CLS], [SEP], [PAD], etc.,) while calculating both weights as previous work suggest that these special tokens are heavily attended in deep heads and might be used as a no-op for attention heads (Clark et al., 2019). Finally, bridging signal is measured as the ratio between $w_{1}$ and $w_{2}$ as mentioned earlier.

\subsection{Experimental Setup}

We provide sentences containing a bridging anaphor (Ana) and its antecedent (Ante) to the pre-trained BERT model as a single sentence without the "[SEP]" token in-between. However, an 
anaphor and its antecedent do not always lie in the same or adjacent sentence(s). Therefore, we design two different experiments. In the first setup, we provide the model with only those sentences which contain Ana and Ante while ignoring all the other sentences in-between. This setting is a bit unnatural as we are not following the original discourse narration. In the second setup, we provide the model with sentences which contain Ana and Ante as well as all the other sentences between Ana and Ante. Note that in both experiments we add markers to denote the anaphor and its antecedent in order to get exact corresponding attention weights.

\subsection{Results With Only Ana-Ante Sentences}

For the input of only sentences containing anaphors and antecedents, we plot the bridging signals corresponding to each attention head separately (see the heatmaps in Figure 1). The left heatmap shows the signals from anaphors to antecedents and the right one shows the signals from antecedents to anaphors. Both heatmaps are based on the pretrained BERT-base-cased model. The $\mathrm{x}$-axis represents the number of attention heads from 1-12 and the $y$-axis represents the number of layers from 112. The darker shade of the color indicates stronger bridging signals and brighter color indicates a weak signal.

The plot shows that the lower layers capture stronger bridging signal in comparison with the middle layers with an exception at the first attention head in the fifth layer. Also, the higher layers pay most attention to bridging relations in comparison to the middle and lower layers. The observation is consistent in both directions - from anaphors to antecedents and from antecedents to anaphors.

\subsection{Results With All Sentences}

As stated earlier, for an anaphor, the antecedent can lie in the same sentence or any previous sentence. This demands a separate investigation of bridging signals depending on the distance (measured in terms of sentences) between anaphors and antecedents. Therefore, we plot bridging signals captured by all attention heads depending on the distance between anaphors and antecedents in Figure 2.

The first plot shows the signals between anaphors and antecedents where the distance between them is 0 (i.e., they occur in the same sentence). The second and the third plots show the bridging signals between anaphors and antecedents in which the anaphor-antecedent sentence distance is 1 and 2, respectively.

In ISNotes, $77 \%$ of anaphors have antecedents occurring in the same or up to two sentences prior to the anaphor. The remaining anaphors have distant antecedents and each distance group only contains a small number of anaphor-antecedent pairs. Therefore, we divide the remaining anaphors into two coarse groups. The plots in Figure 2d and Figure $2 \mathrm{e}$ are plotted by combining anaphorantecedent pairs which are apart by 3 to 5 sentences and 6 to 10 sentences, respectively. Note that we could not plot attention signals for bridging pairs with sentence distance longer than 10 sentences because of the limitation of the input size in BERT.

We observe that, the patterns which are visible with only anaphor-antecedent sentences as the input (Section 4.3) are consistent even with considering all the sentences between anaphors and antecedents. It is clear that higher layers attend more to bridging relations in comparison with lower and middle layers. Also, the lower layers fail to capture bridging signal as the distance between anaphors and antecedents increases. Attention weights assigned by certain attention heads (5:1, 9:12, 11:3 and 12:2-4) are fairly consistent. One more important thing to observe is that as the distance between anaphors and antecedents increases the overall bridging signal decreases. This can be observed by looking at all the heatmaps in Figure 2 as the heatmaps with lower distances are on the darker side.

\subsection{Discussion}

Based on the results from the previous two experiments, we observed that in the pre-trained BERT model, the higher layers pay more attention to bridging relations in comparison with the middle and the lower layers. This observation is in-line with other studies in which the authors found that simple surface features were captured in the lower layers and complex phenomenons like coreference were captured in the higher layers (Jawahar et al., 2019). Also, the overall attention decreases with the increase in the distance between anaphors and antecedents.

We also observed that there are some prominent attention heads which consistently capture bridging relations (5:1, 9:12, 11:3 and 12:2-4). In order to check which bridging relations are easier or harder for these prominent attention heads to capture, we 
The move will make the drug available free of charge for a time to children with the disease and symptoms of advanced infection.

Last year, when the rising Orange River threatened to swamp the course, the same engineers who are pushing back the Atlantic rushed to build a wall to hold back the flood.

At age eight, Josephine Baker was sent by her mother to a white woman's house to do chores in exchange for meals and a place to sleep - a place in the basement with the coal

\section{Difficult Bridging Relations}

In addition, Delmed, which makes and sells a dialysis solution used in treating kidney diseases, said negotiations about pricing had collapsed between it and a major distributor, National Medical Care Inc. Delmed said Robert S. Ehrlich resigned as chairman, president and chief executive.

Mr. Ehrlich will continue as a director and a consultant.

The night the Germans occupied all of France, Baker performed in Casablanca.

The Free French wore black arm bands, and when she sang "J'ai deux amours" they wept.

Ms.Rose is best on the early years and World War II.

In Geneva, however, they supported Iran's proposal because it would have left the Saudi percentage of the OPEC total intact, and increased actual Saudi volume to nearly 5.3M barrels daily from 5M. Some of the proposed modifications since, however, call on Saudi Arabia to "give back" to the production-sharing pool a token 23,000 barrels .

Table 1: Examples of easy and difficult bridging relations for the prominent heads to recognize. Bridging anaphors are typed in boldface, antecedents in underscore.

further investigated qualitatively to identify bridging pairs that get higher or lower attentions in these attention heads. Specifically, we consider pairs which have the bridging signal ratio (defined in Section 4.1) more than $70 \%$ as easier bridging relations for BERT heads to recognize. If the bridging signal ratio is less than $10 \%$, then the corresponding bridging relation is considered as difficult for BERT heads to identify. We list a few easy and difficult examples in Table 1. In general, we observe that semantically closer pairs are easy for prominent heads to identify (e.g., house-basement, disease-infection). On the other hand, pairs that are distant and require more context-dependent as well as common-sense knowledge inference are difficult for the prominent heads to recognize.

\section{Fill-in-the-gap Probing: LMs as Bridging Anaphora Resolvers}

The transformer-based language models are trained with an objective to predict the masked tokens given the surrounding context. Thus, they can also produce a score for a word which can be placed at the masked token in a given sentence. We make use of this property of the language models and propose a novel formulation to understand the bridging anaphora resolution capacity of the pre-trained language models.

\subsection{Of-Cloze Test}

The syntactic prepositional structure ( $X$ of $Y$, such as "the door of house" or "the chairman of company") encodes a variety of bridging relations. Previous work has used this property to design features and develop embedding resources for bridging (Hou et al., 2013; Hou, 2018a,b).

Inspired by this observation, we formulate bridging anaphora resolution as a cloze task. Specifically, given a bridging anaphor and its context, we insert "of [MASK]" after the head word of the anaphor (see Example 1). We then calculate the probability of each candidate to be filled as the mask token. The highest scoring candidate is selected as the predicted antecedent for the anaphor. One of the advantages of our formulation is that we can easily control the scope of the context for each bridging anaphor (e.g., no-context, local context or global context). This allows us to test the effect of different types of context for bridging inference.

(1) Original context: The survey found that over a three-year period $22 \%$ of the firms said employees or owners had been robbed on their way to or from work or while on the job. Seventeen percent reported their customers being robbed.

Cloze test context: The survey found that over a three-year period $22 \%$ of the firms said employees 
or owners had been robbed on their way to or from work or while on the job. Seventeen percent of [MASK] reported their customers being robbed.

\subsection{Experimental Setup}

Recall that in our Of-Cloze test, antecedent candidates are provided and the highest scoring candidate is selected as the predicted antecedent. These candidates are formed by considering mentions which are occuring prior to the anaphor. We design two different experiment sets based on the scope of antecedent candidates and the surrounding context.

Candidates Scope In the first set of experiments, we consider two different sets of antecedent candidates for an anaphor $a$. The first set contains salient and nearby mentions as antecedent candidates. Here, mentions only from the first sentence of the document, previous two sentences preceding $a$ and the sentence containing $a$ are considered as candidates. This setup follows previous work on selecting antecedent candidates (Hou, 2020a). The second set contains all mentions occurring before the anaphor $a$ from the whole document. The second setup of forming antecedent candidates is more challenging than the first one because the number of candidates increases which makes selecting the correct antecedent difficult.

Next, we provide the same context for anaphors in both of the experiments described above. We construct the context $c$ for the bridging anaphor $a$. Precisely, $c$ contains the first sentence of the document, the previous two sentences occurring before $a$, as well as the sentence containing $a$. We replace the head of $a$ as "of [MASK]".

We also compare this fill-in-the-gap probing approach with the attention heads-based approach for resolving bridging anaphors. Specifically, we use the prominent heads in BERT for identifying bridging relations from Section 4. Here, we obtained attention weights from an anaphor head to all antecedent candidate heads by adding attentions from prominent heads 5:1, 9:12,11:3, and 12:2-4. Then the highest scoring candidate is predicted as the antecedent for the anaphor.

Context Scope In the second set of experiments, we concentrate on probing the behavior of language models at capturing bridging relations with different contexts. We experiment with the following four settings:

- a. Only anaphor: in this setup, only the anaphor phrase (with "of [MASK]" being inserted after the anaphor's head word) is given as the input to the model.

- b. Anaphor sentence: the sentence containing the anaphor is provided. The phrase "of [MASK]" is inserted after the head word of the anaphor.

- c. Ante+Ana sentence: on top of b, the sentence containing the antecedent is also included in the context.

- d. More context: on top of b, the first sentence from the document as well as the previous two sentences preceding the anaphor are included.

Without "of" Context To test the effect of the strong bridging indicating signal "of", we further execute another set of experiments. Specifically, We remove "of" from "anaphor ${ }_{\text {head }}$ of [MASK]" and instead, provide "anaphor ${ }_{\text {head }}$ [MASK]" for each type of the context described above.

Perturbed Context In this setting, we perturb the context by randomly shuffling the words in the context except for the anaphor and antecedent phrases for each type of the context mentioned above. Note that we still have the "of" indicator in this setup.

\subsection{Results and Discussion}

\subsubsection{Results on Candidates Scope}

Table 2 shows the accuracy of using only the prominent heads and our Of-Cloze test approach for bridging anaphora resolution. All experiments are based on the same context (i.e., the sentence containing an anaphor, the previous two sentences preceding the anaphor as well as the first sentence from the document).

We find that the Of-Cloze probing approach achieves higher result in comparison to the prominent attention head approach (31.64\% vs. $20.15 \%)$ under the same conditions. One reason might be that although other attention heads do not significantly attend to bridging relations but cumulatively they are effective.

We also observe that in the Of-Cloze test, the results of using salient/nearby mentions as antecedent candidates are better than choosing antecedents from all previous mentions (Row (2) vs. Row (3), and Row (2) vs. Row (4)). This is because the model has to choose from a smaller number of candidates in the first case as the average number of 


\begin{tabular}{|l|l|l|l|l|c|}
\hline $\begin{array}{l}\text { Antecedent } \\
\text { Candidate Scope }\end{array}$ & $\begin{array}{l}\text { No. } \\
\text { Anaphors }\end{array}$ & $\begin{array}{l}\text { BERT- } \\
\text { Base }\end{array}$ & $\begin{array}{l}\text { BERT- } \\
\text { Large }\end{array}$ & $\begin{array}{l}\text { RoBERTa- } \\
\text { Base }\end{array}$ & $\begin{array}{l}\text { RoBERTa- } \\
\text { Large }\end{array}$ \\
\hline \multicolumn{7}{|c|}{ Prominent attention heads } \\
\hline (1) Salient/nearby mentions & 531 & 20.15 & - & - & - \\
\hline \multicolumn{7}{|c|}{ Of-Cloze Test } \\
\hline (2) Salient/nearby mentions & 531 & 31.64 & 33.71 & 34.08 & $\mathbf{3 4 . 6 5}$ \\
\hline (3) All previous mentions & 622 & 26.36 & 28.78 & 27.49 & $\mathbf{2 9 . 9 0}$ \\
\hline \multicolumn{7}{|c|}{ Of-Cloze Test: Anaphors with antecedents in the provided contexts } \\
\hline (4) All previous mentions & 531 & 29.00 & 30.88 & 30.32 & $\mathbf{3 2 . 3 9}$ \\
\hline \multicolumn{7}{|c|}{ Of-Cloze Test: Anaphors with antecedents outside of the provided contexts } \\
\hline (5) All previous mentions & 91 & 10.98 & $\mathbf{1 6 . 4 8}$ & 10.98 & 15.38 \\
\hline
\end{tabular}

Table 2: Result of selecting antecedents for anaphors with two different probing approaches (Prominent attention heads and Of-Cloze Test) based on the same context. Accuracy is calculated over a different number of anaphors.

\begin{tabular}{cc}
\hline Distance & Accuracy \\
\hline salient* $^{*}$ & 38.65 \\
0 & 26.92 \\
1 & 20.58 \\
2 & 17.30 \\
$>2$ & 10.98 \\
\hline
\end{tabular}

Table 3: Anaphor-antecedent distance-wise accuracy with the BERT-base-cased model. * indicates that the antecedent is in the first sentence of the document.

antecedent candidates are only 22 per anaphor as opposed to 148 in the later case.

We further divide 622 anaphors in Row (3) into two groups (Row (4) and Row (5) in Table 2) depending on whether the corresponding antecedents occur in the provided contexts. It can be seen that the performance is significantly better when antecedents occur in the contexts.

Finally, when comparing the results of each language model in each row separately, it seems that the bigger models are always better at capturing bridging information. In general, the RoBERTalarge model performs better than other models except when antecedents do not occur in the provided contexts (Row (5)).

Note that the results in Table 2 are not calculated over all 663 anaphors in ISNotes. Therefore, if the results are normalized over all anaphors then we get the best result with the RoBERTa-large model $(28.05 \%)$, which is reasonably fine in comparison with the state-of-the-art result of $50.08 \%$ (Hou, 2020a) given that the model is not fine-tuned for the bridging task.

\begin{tabular}{llll}
\hline Context Scope & $\begin{array}{l}\text { with } \\
\text { "of" }\end{array}$ & $\begin{array}{l}\text { without } \\
\text { "of" }\end{array}$ & perturb \\
\hline only anaphor & 17.20 & 5.62 & - \\
ana sent. & 22.82 & 7.71 & 10.28 \\
ana+ante sent. & 27.81 & 9.61 & 10.93 \\
more context & 26.36 & 12.21 & 11.41 \\
\hline
\end{tabular}

Table 4: Accuracy of selecting antecedents with different types of context using BERT-of-Cloze Test.

\subsubsection{Results on Ana-Ante Distance}

We further analyze the results of choosing antecedents obtained using the BERT-base-cased model with all previous mentions as the antecedent candidate scope in our Of-Cloze test probing experiment (Row (3) in Table 2) to understand the effect of distance between anaphors and antecedents. The results are shown in Table 3.

In general, it seems that the accuracy decreases as the distance between anaphors and antecedents increases except when antecedents are from the first sentences of the documents. This is related to the position bias in news articles from ISNotes. Normally globally salient entities are often introduced in the beginning of a new article and these entities are preferred as antecedents.

The other reason for the lower results in case of antecedents being away for more than two sentences might be that these antecedents are absent from the provided context.

\subsubsection{Results on Different Contexts}

The results of experiments with different types of context are shown in Table 4. All experiments are based on the BERT-base-cased model with all pre- 
vious mentions as the antecedent candidate scope We refer to this model as BERT-Of-Cloze in the following discussion.

In the first column of the table, BERT-Of-Cloze achieves an accuracy score of $17.20 \%$ with only the anaphor information plus "of [mask]". We can see that the results improve incrementally with the addition of context. More specifically, the accuracy score improves from $17.20 \%$ to $22.82 \%$ by adding sentences containing anaphors. Adding sentences which contain antecedents (ana + ante sent.) further improves the accuracy score to $27.81 \%$. Finally, adding more local context and the first sentence leads to an accuracy score of $26.36 \%$. Note that compared to "ana + ante sent.", "more context" represents a more realistic scenario in which we do not assume that the antecedent position information is known beforehand. In general, the results in the first column of Table 4 indicate that the model can leverage context information when predicting antecedents for bridging anaphors.

Results reduce drastically when "of" is removed from the "anaphor of [MASK]" phrase (Table 4, column:2) from all context scopes. Without this indicator, the language model cannot make sense of two adjacent tokens such as "consultant company".

It is interesting to see that the results reduced drastically as well when we perturb the context between the anaphor and antecedent (Table 4, last column). This establishes the importance of meaningful context for performing bridging inference effectively in transformer language models.

\subsection{Error Analysis: Of-Cloze test}

We analyzed anaphor-antecedent pairs that are linked wrongly by the $O f$-Cloze formulation and observed some common erros.

Failure at capturing sophisticated commonsense knowledge: We found that the pre-trained transformer language model such as BERT acquires simple common-sense knowledge, therefore it can link anaphor-antecedent pairs such as "sand-dunes" and "principal-school". But it fails at capturing sophisticated knowledge, such as "consultant-Delmed (a company)" and "poolOPEC (Organization of petroleum countries)". This might be happening because of the rare cooccurrences of these pairs in the original text on which BERT is pre-trained. Also, BERT has inherent limitations at acquiring such structured knowledge (Park et al., 2020).
Language modelling bias: In our $O f$-Cloze test probing, we use pre-trained transformer language models without fine-tuning. As a result, the model fills masked tokens that are fit according to the language modeling objective, not for bridging resolution. Thus, sometimes, the selected token perfectly makes sense in the single sentence but the choice is incorrect in the broader context. Consider the example, "Only $22 \%$ of [MASK] supported private security patrols $[\ldots]$ ". BERT predicts "police" as a suitable antecedent that produces a meaningful local sentence. However, the correct antecedent is "correspondents" according to the surrounding context of this sentence.

Unsuitable formulation for set-relations: Our Of-Cloze formulation produces awkward phrases for some bridging pairs that possess set-relations. Considering a bridging pair - "One man - employees", in this case the model should assign high score for the phrase - "One man of employees". But, as this phrase is quite clumsy, BERT naturally being a language model assigns low scores for these pairs.

\section{Conclusions}

We investigated the effectiveness of pre-trained transformer language models in capturing bridging relation inference by employing two distinct but complementary approaches.

In the first approach, we probed individual attention heads in BERT and observed that attention heads from higher layers prominently captured bridging compared to the middle and lower layers and some specific attention heads consistently looked for bridging relation. In our second approach, we considered using language models for bridging anaphora resolution by formulating the task as a $O f$-Cloze test. We carefully designed experiments to test the influence of different types of context for language models to resolve bridging anaphors. Our results indicate that pre-trained transformer language models encode substantial information about bridging.

Finally, in this work, we only focus on understanding the capacity of the pre-trained language models for bridging inference. Based on the insights we gained from the current probing study, in the future, we plan to explore how to better use pretrained transformer language models for bridging resolution. 


\section{Acknowledgements}

We thank the three anonymous reviewers for their comments and feedback. This work was partially supported by the French National Research Agency via grant no ANR-16-CE33-0011-01 as well as by CPER Nord-Pas de Calais/FEDER DATA Advanced data science and technologies 2015-2020.

\section{References}

Nicholas Asher and Alex Lascarides. 1998. Bridging. Journal of Semantics, 15:83-113.

Johan Bos, Paul Buitelaar, and Anne Marie Mineur. 1995. Bridging as coercive accomodation. In Working Notes of the Edinburgh Conference on Computational Logic and Natural Language Processing (CLNLP-95), Human Communications Research Centre, University of Edinburgh, Edinburgh, U.K.

Herbert H. Clark. 1975. Bridging. In Proceedings of the Conference on Theoretical Issues in Natural Language Processing, Cambridge, Mass., June 1975, pages 169-174.

Kevin Clark, Urvashi Khandelwal, Omer Levy, and Christopher D. Manning. 2019. What does BERT look at? an analysis of BERT's attention. In Proceedings of the 2019 ACL Workshop BlackboxNLP: Analyzing and Interpreting Neural Networks for NLP, pages 276-286, Florence, Italy. Association for Computational Linguistics.

Jeff Da and Jungo Kasai. 2019. Cracking the contextual commonsense code: Understanding commonsense reasoning aptitude of deep contextual representations. In Proceedings of the First Workshop on Commonsense Inference in Natural Language Processing, pages 1-12, Hong Kong, China. Association for Computational Linguistics.

Jacob Devlin, Ming-Wei Chang, Kenton Lee, and Kristina Toutanova. 2019. BERT: Pre-training of deep bidirectional transformers for language understanding. In Proceedings of the 2019 Conference of the North American Chapter of the Association for Computational Linguistics: Human Language Technologies, Volume 1 (Long and Short Papers), pages 4171-4186, Minneapolis, Minnesota. Association for Computational Linguistics.

Yoav Goldberg. 2019. Assessing BERT's syntactic abilities. ArXiv, abs/1901.05287.

Yufang Hou. 2018a. A deterministic algorithm for bridging anaphora resolution. In Proceedings of the 2018 Conference on Empirical Methods in Natural Language Processing, pages 1938-1948, Brussels, Belgium. Association for Computational Linguistics.
Yufang Hou. 2018b. Enhanced word representations for bridging anaphora resolution. In Proceedings of the 2018 Conference of the North American Chapter of the Association for Computational Linguistics: Human Language Technologies, Volume 2 (Short Papers), pages 1-7, New Orleans, Louisiana. Association for Computational Linguistics.

Yufang Hou. 2020a. Bridging anaphora resolution as question answering. In Proceedings of the 58th Annual Meeting of the Association for Computational Linguistics, pages 1428-1438, Online. Association for Computational Linguistics.

Yufang Hou. 2020b. Fine-grained information status classification using discourse context-aware BERT. In Proceedings of the 28th International Conference on Computational Linguistics, Barcelona, Spain. Association for Computational Linguistics.

Yufang Hou, Katja Markert, and Michael Strube. 2013. Global inference for bridging anaphora resolution. In Proceedings of the 2013 Conference of the North American Chapter of the Association for Computational Linguistics: Human Language Technologies, pages 907-917, Atlanta, Georgia. Association for Computational Linguistics.

Phu Mon Htut, Jason Phang, Shikha Bordia, and Samuel R. Bowman. 2019. Do attention heads in bert track syntactic dependencies? ArXiv, abs/1911.12246.

Sarthak Jain and Byron C. Wallace. 2019. Attention is not Explanation. In Proceedings of the 2019 Conference of the North American Chapter of the Association for Computational Linguistics: Human Language Technologies, Volume 1 (Long and Short Papers), pages 3543-3556, Minneapolis, Minnesota. Association for Computational Linguistics.

Ganesh Jawahar, Benoît Sagot, and Djamé Seddah. 2019. What does BERT learn about the structure of language? In Proceedings of the 57th Annual Meeting of the Association for Computational Linguistics, pages 3651-3657, Florence, Italy. Association for Computational Linguistics.

Olga Kovaleva, Alexey Romanov, Anna Rogers, and Anna Rumshisky. 2019. Revealing the dark secrets of BERT. In Proceedings of the 2019 Conference on Empirical Methods in Natural Language Processing and the 9th International Joint Conference on Natural Language Processing (EMNLP-IJCNLP), pages 4365-4374, Hong Kong, China. Association for Computational Linguistics.

Katja Markert, Yufang Hou, and Michael Strube. 2012. Collective classification for fine-grained information status. In Proceedings of the 50th Annual Meeting of the Association for Computational Linguistics (Volume 1: Long Papers), pages 795-804, Jeju Island, Korea. Association for Computational Linguistics. 
S. Park, J. Son, S. w. Hwang, and K. Park. 2020. Bert is not all you need for commonsense inference. In ICASSP 2020 - 2020 IEEE International Conference on Acoustics, Speech and Signal Processing (ICASSP), pages 8204-8208.

Fabio Petroni, Tim Rocktäschel, Sebastian Riedel, Patrick Lewis, Anton Bakhtin, Yuxiang Wu, and Alexander Miller. 2019. Language models as knowledge bases? In Proceedings of the 2019 Conference on Empirical Methods in Natural Language Processing and the 9th International Joint Conference on Natural Language Processing (EMNLPIJCNLP), pages 2463-2473, Hong Kong, China. Association for Computational Linguistics.

Ina Roesiger, Arndt Riester, and Jonas Kuhn. 2018. Bridging resolution: Task definition, corpus resources and rule-based experiments. In Proceedings of the 27th International Conference on Computational Linguistics, pages 3516-3528, Santa Fe, New Mexico, USA. Association for Computational Linguistics.

Ionut-Teodor Sorodoc, Kristina Gulordava, and Gemma Boleda. 2020. Probing for referential information in language models. In Proceedings of the 58th Annual Meeting of the Association for Computational Linguistics, pages 4177-4189, Online. Association for Computational Linguistics.

Alon Talmor, Yanai Elazar, Yoav Goldberg, and Jonathan Berant. 2020. oLMpics - on what language model pre-training captures. Transactions of the Association for Computational Linguistics, 8.

Thomas Wolf, Lysandre Debut, Victor Sanh, Julien Chaumond, Clement Delangue, Anthony Moi, Pierric Cistac, Tim Rault, Rémi Louf, Morgan Funtowicz, Joe Davison, Sam Shleifer, Patrick von Platen, Clara Ma, Yacine Jernite, Julien Plu, Canwen $\mathrm{Xu}$, Teven Le Scao, Sylvain Gugger, Mariama Drame, Quentin Lhoest, and Alexander M. Rush. 2020. Transformers: State-of-the-art natural language processing. In Proceedings of the 2020 Conference on Empirical Methods in Natural Language Processing: System Demonstrations, pages 38-45, Online. Association for Computational Linguistics. 